\title{
Delimitation of the Gametangia of Rhizopus sexualis (Smith) Callen: an Electron Microscope Study of Septum Formation
}

\author{
By LILIAN E. HAWKER AND MARGARET A. GOODAY \\ Department of Botany, University of Bristol \\ (Accepted for publication 30 May 1967)
}

SUMMARY

The development of the septa which cut off the gametangia of Rhizopus sexualis from the suspensor cells was studied by electron microscopy. The edge of the ingrowing septum extends by the incorporation of coalesced vesicles which were previously aligned in the plane of the developing septum. The membranes surrounding these vesicles fuse to form the plasmalemmas of the gametangium and suspensor cell and are eventually continuous with the plasmalemma of the original progametangium. New wall material is laid down between these plasmalemmas. Associated with this process is a considerable increase in complexity of the endoplasmic reticulum which forms broad, irregular bands on either side of the septum. Fine tubules, resembling plasmodesmata, pass through the wall at intervals. Thickening of the septum proceeds more rapidly on the gametangial side.

\section{INTRODUCTION}

Soon after a pair of progametangia of Rhizopus sexualis make contact at their apices, the terminal parts (gametangia) become cut off from the suspensor cells by the formation of transverse septa. This paper describes the process of formation of these septa as revealed by the electron microscope.

\section{METHODS}

The fungus was grown on cellophane laid on the surface of malt-agar plates. Young colonies with all stages of zygospore development present were fixed in $2 \%(\mathrm{w} / \mathrm{v}$ unbuffered $\mathrm{KMnO}_{4}$ for $30 \mathrm{~min}$. at room temperature, and washed several times in distilled water. The hyphae became brittle during fixation and the zygospores were readily detached from the mycelium during washing. Zygospores were then collected in centrifuge tubes, dehydrated, and embedded in Epon or Vestopal. Sections were cut at approximately $450-550 \AA$ thickness.

\section{RESULTS}

The first sign of septum development detectable by light microscopy is a slight decrease in density of the cell contents on the inner side of the enveloping walls of the progametangium at the site from which the septum would be expected to develop. The electron microscope revealed, however, that in such a specimen a thin wall (about I350 $\AA$ thick at the junction with the progametangial wall and tapering to about $385 \AA$ thickness at the advancing inner edge) was already partially developed, extending inwards from the peripheral wall and leaving a central gap of about one third of the 
total diameter of the progametangium (Fig. I $a, b$ ). At this stage the septum was not set at right angles to the original enveloping progametangial wall but formed an acute angle with it growing obliquely inwards in a direction away from the apex of the progametangium (Fig. I $b$; Pl. I, fig. I). Sections through the developing septum gave a different picture of the advanding edge according to whether they were radial or tangengial longitudinal sections of the zygospore initial (Fig. I $a-c$; Pl. I, fig. 2; Pl. 2, figs. $3-5$ ).

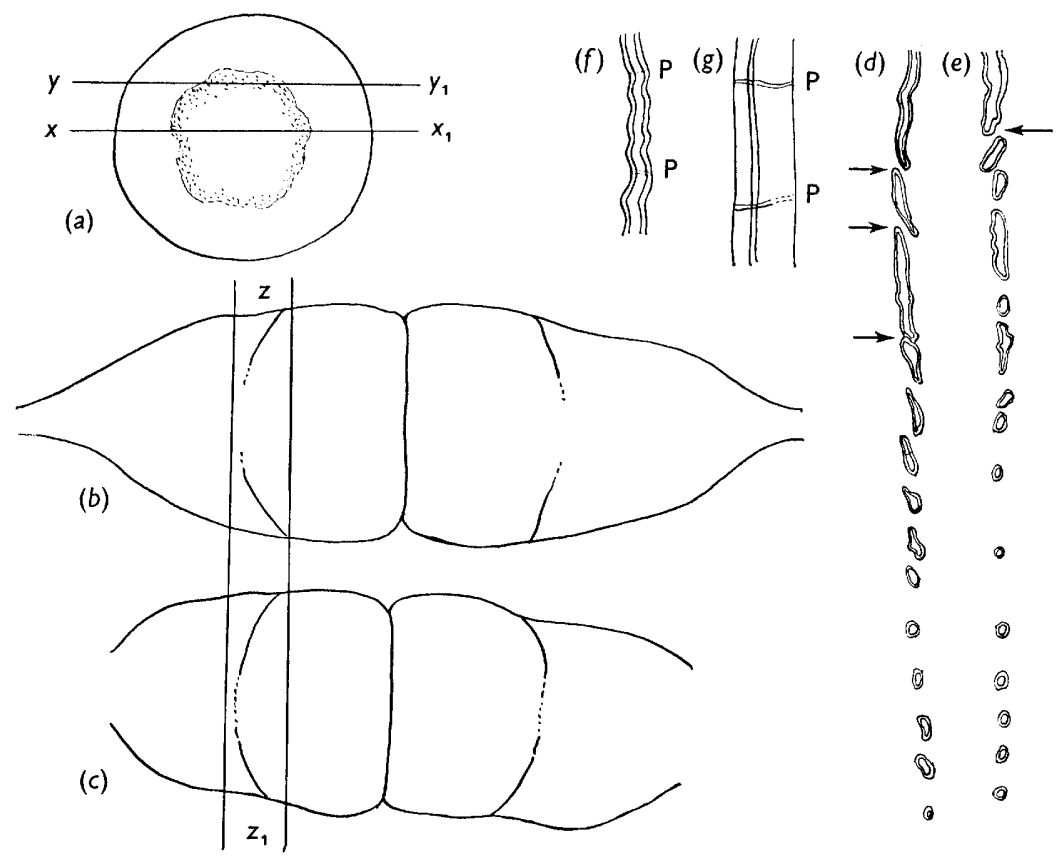

Fig. I. $a-c$, Diagrammatic $(\times 500) ; d-g$, drawn to scale from electronmicrographs $(\times 20,000)$. (a) Developing septum as it would be seen in optical surface view (i.e. in transverse section of zygospore shown as zone $z-z_{1}$ in Fig. $1 b, c$ ), inner edge consisting of disconnected vesicles arranged in plane of septum. (b) Radial longitudinal section of zygospore through plane $\left(x-x_{1}\right)$ of $(a)$, showing developing septum set at an angle to progametangial wall and curving towards the centre of the progametangium, thus bringing the advancing edges into the same plane. A band of disconnected vesicles continues the plane of the septum inwards for a short distance. (c) Tangential longitudinal section of same zygospore through plane $\left(y-y_{1}\right)$ of $(a)$, showing disconnected vesicles extending across the gap between the advancing edge of the septum. $(d, e)$. Edges of septum as seen in longitudinal radial and tangential sections respectively. Note overlapping of some of newly added, but as yet incompletely fused, sections of wall (indicated by arrows) resulting from imprecise alignment of contributing vesicles. $(f)$ Section through older part of septum; irregularities due to overlapping of contributing segments now visible only as undulation of wall. $(g)$ Older septum, secondary thickening beginning, undulations now barely visible. Note plasmodesmata (P).

The most recently formed part of the septum was very thin and undulating. It consisted of an inner electron-transparent zone or plate, sandwiched between two electrondense layers (Pl. 2, figs. 3,6). The line of the wall, as seen in radial section, was continued inwards for a short distance (P1. 2, fig. 4) as a row of unconnected vesicles. Further towards the centre of the cell there was a broader band extending in the same plane as the advancing wall, but consisting of unaligned vesicles, circular or elongated as seen in section. These might have been spherical vesicles and tubules, respectively, 
or part of a reticular network. Associated with these were several globules of regular outline, enveloped by a single membrane and containing an unknown substance or substances (Pl. 2, figs. 3, 4).

In tangential sections obviously cut near and just within the advancing edge of the septum (Fig. I $a$ ) the line of vesicles, as would be expected, was continuous across the central gap (Pl. I, fig. 2). It can be seen that these 'vesicles' tended to coalesce and that this tendency was greatest adjacent to the developing septum (P1. 2, fig. 3). Here they joined to form plates (often at first slightly overlapping like roof tiles, Fig. I $d, e$ ) which finally coalesced to give a continuous septum. It is clear that the septum developed by the addition to its edge of coalesced cisternae or vesicles which had already become aligned in the plane of the septum. This method of wall formation through the coalescence of vesicles was reflected in the wavy outline of the newly formed wall (as seen in P1. 2, fig. 6; Fig. If), which persisted until the wall began to thicken. Finally the wall extended across the progametangium, leaving no central pore.

The membranes surrounding the vesicles which became incorporated in the septum became reorientated and formed two parallel layers comparable with and finally continuous with the original plasmalemma of the progametangium (Pl. I, fig. I). As the septum developed, wall material similar in appearance to that of the original progametangial wall was laid down between the two parallel plasmalemma membranes (PI. 3, fig. 7), beginning at the peripheral zone (i.e. adjacent to the progametangial wall) of the new wall and proceeding inwards so that the developing septum was a narrowbased wedge, as seen in section. At a very early stage it could be seen that the new wall material was laid down immediately within the parallel plasmalemma membranes and that there was a thin electron-transparent central zone, corresponding to the original interior of the component coalesced vesicles and separating the two new layers of wall material (Pl. 2, fig. 6). At this early stage the two layers of new wall material were of approximately equal thickness. At intervals along the septum transverse fine tubules could be seen, recalling the plasmodesmata of higher plant cells (Pl. 3, fig. 7). These have already been reported in this fungus and in Gilbertella persicaria by Hawker, Gooday \& Bracker (I966). At about this stage the endoplasmic reticulum, which had previously been sparsely but fairly evenly distributed throughout the progametangial cytoplast (as is typical of fungus cells; Hawker, 1965), became aggregated into complex masses along the septum on both sides of the newly formed wall (Pl. 3, figs. 8, 9) beginning in the angle between the septum and the original progametangial wall (P1. 3, fig. 7). The septum then rapidly thickened by the deposition of new layers of wall material, presumably adjacent to the plasmalemma. This process, however, was much more rapid and continued longer on the gametangial side of the wall (P1. 3, fig. 9), where it eventually formed part of the thick wall of the zygospore.

Despite this uneven rate of thickening of the wall layers on the gametangial and suspensor sides of the septum, no differences in the nature or amount of the adjacent bands of endoplasmic reticulum were detected. The plasmodesmata-like tubules could be seen passing across the entire width of the septum (P1. 3, fig. 9) and may well function as conducting channels to allow passage of soluble materials through the developing wall.

The development of the two gametangia is not usually exactly synchronous in a conjugating pair, so that the same zygospore initial may show septa at different stages of development. 


\section{DISCUSSION}

Detailed electron-microscope studies of septum formation are available for only a few organisms. Bracker (I966) described the formation of sporangiospores of Gilbertella persicaria (Eddy) Hesseltine. Here the spores were delimited and separated by the fusion of cleavage vesicles which developed between the spore initials. The membranes of these cleavage vesicles became the plasmalemma of the young spores. The spore envelope and, later, secondary wall layers, were formed outside the plasmalemma (i.e. on the side of the vesicle membrane which was originally innermost), as with the gametangial septum of Rhizopus. The characteristic dark dots associated with these membranes in Gilbertella were not seen in Rhizopus. Bracker reported that endoplasmic reticulum aggregates were often associated with the cleavage vesicles, but his photographs, which related to comparatively early stages of sporangiospore formation, do not show such large complex aggregations as those which developed along the gametangial wall of the Rhizopus zygospore.

Bracker pointed out that wall formation of ascospores (Moore, I963; Moore \& McAlear, 1962; Wilsenach \& Kessel, 1965) differs in some essential points from sporangiospore formation; it also bears little resemblance to septum formation in the progametangium of Rhizopus. The closest parallel to the latter is the formation of septa in higher plant cells. Whaley, Dauwalder \& Kephart (1966) illustrated the formation of a septum across the spindle plate of a dividing root cell of maize (Zea mays L.). Here the septum, in contrast to that of Rhizopus, begins at the centre of the cell and develops outwards, but it extends by the incorporation of coalesced vesicles in a manner essentially similar to that described above (p. 373). Frey-Wyssling, López-Saéz \& Mühlethaler (1964) showed a similar type of wall development in Phalaris canariensis $\mathbf{L}$.

In the higher plant, septum formation is associated with the presence of dictyosomes (Golgi apparatus). Mollenhauer, Whaley \& Leech (I96I) and Leech, Mollenhauer \& Whaley (I963) considered that the Golgi bodies contribute not only to plate formation but also to later stages of wall development. No dictyosomes or similar regularly arranged plates of cisternae were seen during septum formation of Rhizopus. It was shown, however, that the vesicles which became integrated with the advancing edge of the septum arose in an area comparatively rich in irregular vesicles and cisternae, and that as the wall began to thicken a very great increase in endoplasmic reticulum in the form of tangled tubules occurred. Such complexes most probably play a part in wall formation similar to that of the dictyosomes. Porter (I96I) in a discussion of the function of endoplasmic reticulum concluded that at the cell surface the units of the reticulum are short or circular, as seen in section, suggesting a tubular form rather than the typical lamellar form. He considered that the functional activity of these structures at the cell surface includes 'some exchange of metabolites and the production and deposition of materials in wall formation'. It is reasonable to conclude that the striking increase in complexity and amount of endoplasmic reticulum in irregular zones along both sides of the gametangial septum of Rhizopus is similarly associated with deposition of wall material and that exchange of metabolites between suspensor and gametangium may take place to a limited extent via the tubules seen to extend through the septum and which remain clearly visible and apparently unoccluded until at least a late stage in secondary wall thickening. 
Wilsenach \& Kessel (1965) considered that lomasomes (first thought to be peculiar to fungi; Moore \& McAlear, I96I) play an important part in the formation of the ascospore wall in Penicillium vermiculatum Dangeard. These authors described the origin of lomasomes between the membranes of endoplasmic vesicles which were associated with the ascospore wall. Lomasomes have recently been seen in organisms other than fungi and have been shown to consist of aggregates of fine tubules (Hendy, 1966). They occur occasionally adjacent to the development gametangial septum of Rhizopus and may perhaps be interpreted as extracytoplasmic tubules essentially similar in function to those within the cytoplasm.

This study of zygospore development in Rhizopus is being continued by electron microscopy and by differential staining; it is hoped that it will further illuminate the various processes which lead to the production of mature zygospores.

Thanks are due to the Science Research Council for a grant to L.E.H. in aid of this investigation which forms part of a larger project.

\section{REFERENCES}

BRACKER, C. E. (I966). Ultrastructural aspects of sporangiospore formation in Gilbertella persicaria. In The Fungus Spore. Ed. by M. F. Madelin. Colston Pap. 18, 39.

Frey-Wyssling, A., López-SaÉz, J. F. \& Mühlethaler, K. (1964). Formation and development of the cell plate. $J$. ultrastruct. Res. ro, 422 .

HAWKer, L. E. (I965). Fine structure of fungi as revealed by electron microscopy. Biol. Rev. 40, 59. Hawker, L. E., GoOday, M. A. \& Bracker, C. E. (1966). Plasmodesmata in fungal cell walls. Nature, Lond. 212, 635.

Hendy, R. J. (1966). Resemblance of lomasomes of Pythium debaryanum to structures recently described in Chara and Nitella. Nature, Lond. 209, 1258.

Leech, J. H., Mollenhauer, H. H. \& Whaley, J. H. (I963). Ultrastructural changes in the root apex. Symp. Soc. exp. Biol. 17, 74.

Mollenhauer, H. H., Whaley, J. H. \& Leech, J. H. (196r). A function of the Golgi apparatus in outer rootcap cells. J. ultrastruct. Res. 5, 193.

Moore, R. T. (1963). Fine structure of mycota. I. Electron microscopy of the Discomycete Ascodesmis. Nova Hedwigia 5, 263.

Moore, R. T. \& McAlear, J. H. (196I). Fine structure of mycota. V. Lomasomes; previously uncharacterized hyphal structures. Mycologia 55, 633 .

Moore, R. T. \& MCAlEAR, J. H. (I962). Observations on ascospore initiation in the Discomycete Dasyscyphus. J. gen. Microbiol. 28, 21 I.

PORTER, K. R. (I96I). The endoplasmic reticulum: some current interpretation of its forms and functions. In Biological Function and Form. Ed. by O. Lindberg, and T. W. Goodwin. Proc. Ist IUB/IUBS Int. Symp., Stockholm.

ReYNOLDS, E. S. (I963). The use of lead citrate at a high $\mathrm{pH}$ as an electron opaque stain in electron microscopy. J. cell. Biol. 17, 208.

Whaley, W. G., Dauwalder, M. \& Kephart, J. E. (I966). The Golgi apparatus and an early stage in cell plate formation. J. ultrastruct. Res. $\mathbf{1 5}, 169$.

WilsENACH, R. \& KeSSEL, M. (1965). The role of lomasomes in wall formation in Penicillium vermiculatum. J. gen. Microbiol. 40, 40I. 


\section{EXPLANATION OF PLATES}

Key to symbols: $\mathrm{S}=$ suspensor cell, $\mathrm{G}=$ gametangium, $\mathrm{PW}=$ original progametangial wall, $\mathrm{CW}=$ new cross wall (septum), $\mathrm{M}=$ mitochondrion, $\mathrm{N}=$ nucleus, $\mathrm{SV}=$ storage vacuole, $\mathbf{P}=$ plasmodesmata, $\mathrm{V}=$ vesicle, $\mathrm{ER}=$ endoplasmic reticulum. Lines on figures represent $\mathrm{I} \mu$ in each case.

Figs. $\mathrm{I}-7$ were fixed in $2 \% \mathrm{KMnO}_{4}, 30$ min., soaked in $0.5 \%$ Aq uranyl acetate 48 min., dehydrated, embedded in Epon; sections stained ro min. in lead citrate (Reynolds, 1963). Fig. 8 was fixed in $2 \% \mathrm{KMnO}_{4}, 30$ min., dehydrated, embedded in Epon; sections stained lead citrate. Fig. 9. as Fig. 8, but embedded in Vestopal.

\section{Plate I}

Fig. I. Radial longitudinal section through incomplete septum. Note angle with progametangial wall, P.W.; continuity of plasmalemma of original cell with outer membrane of new wall; deposition of new secondary wall material (at part of septum adjacent to progametangial wall) between plasmalemma and central electron-transparent plate of septum; aggregation of endoplasmic reticulum (ER) in angle between septum and original wall; and two vesicles (V) aligned in plane of advancing edge of septum.

Fig. 2. Tangential longitudinal section through same developing septum (see Text-fig. I). Note line of coalescing vesicles in plane of septum.

\section{Plate 2}

Fig. 3. A similar section to that of Fig. 2, showing details of the joining together of coalesced vesicles to form the advancing edge of the septum.

Fig. 4. A more nearly median section through the edge of the septum and part of the central gap showing a zone of irregular ER from which the wall-forming vesicles are budded off.

Fig. 5. Part of fig. 4 enlarged.

Fig. 6. Part of the newly developed septum, before secondary thickening, showing plasmalemma, two adjacent 'grey' zones and the central electron transparent one. Note occasional 'plasmodesmata' (P).

\section{Plate 3}

Fig. 7. Section through junction of septum and lateral wall showing increased thickness at outer part due to secondary deposition of new wall material. Note also plasmodesmata $(P)$.

Fig. 8. Similar section of septum at slightly older stage, showing unequal thickening, greater on gametangial side.

Fig. 9. Later stage, showing pronounced thickening on gametangial side of septum. Plasmodesmata clearly seen as fine tubules passing through wall. 

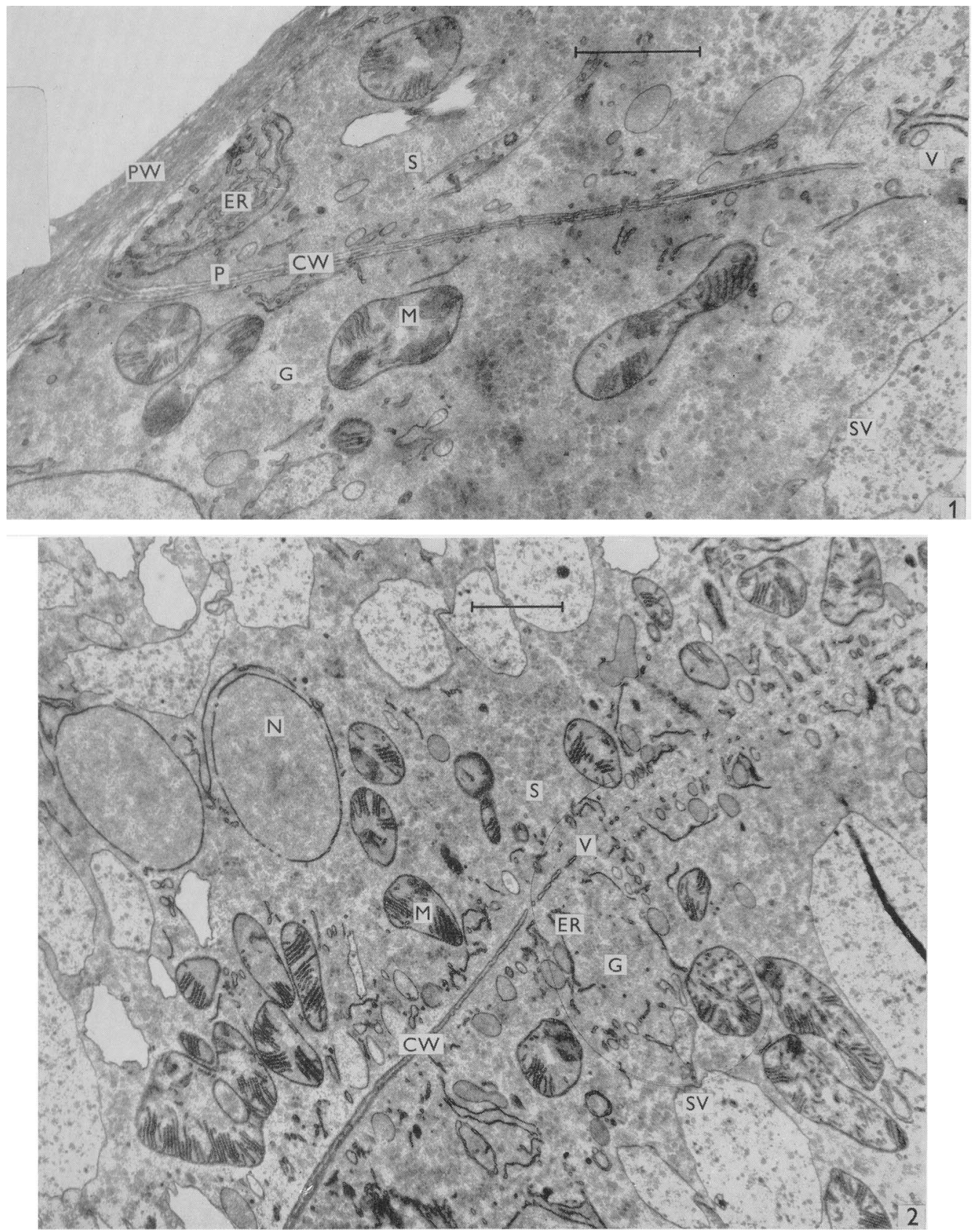

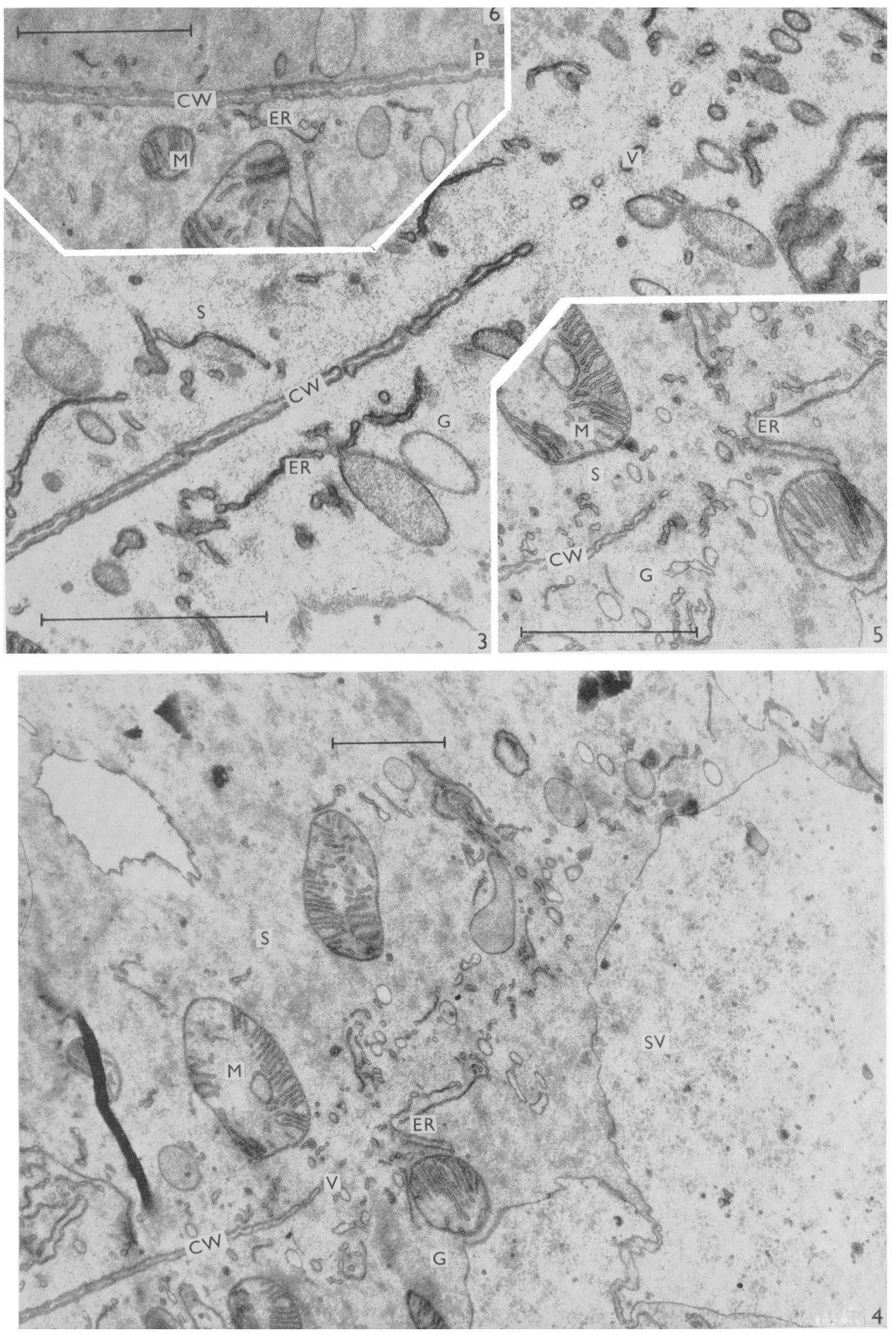


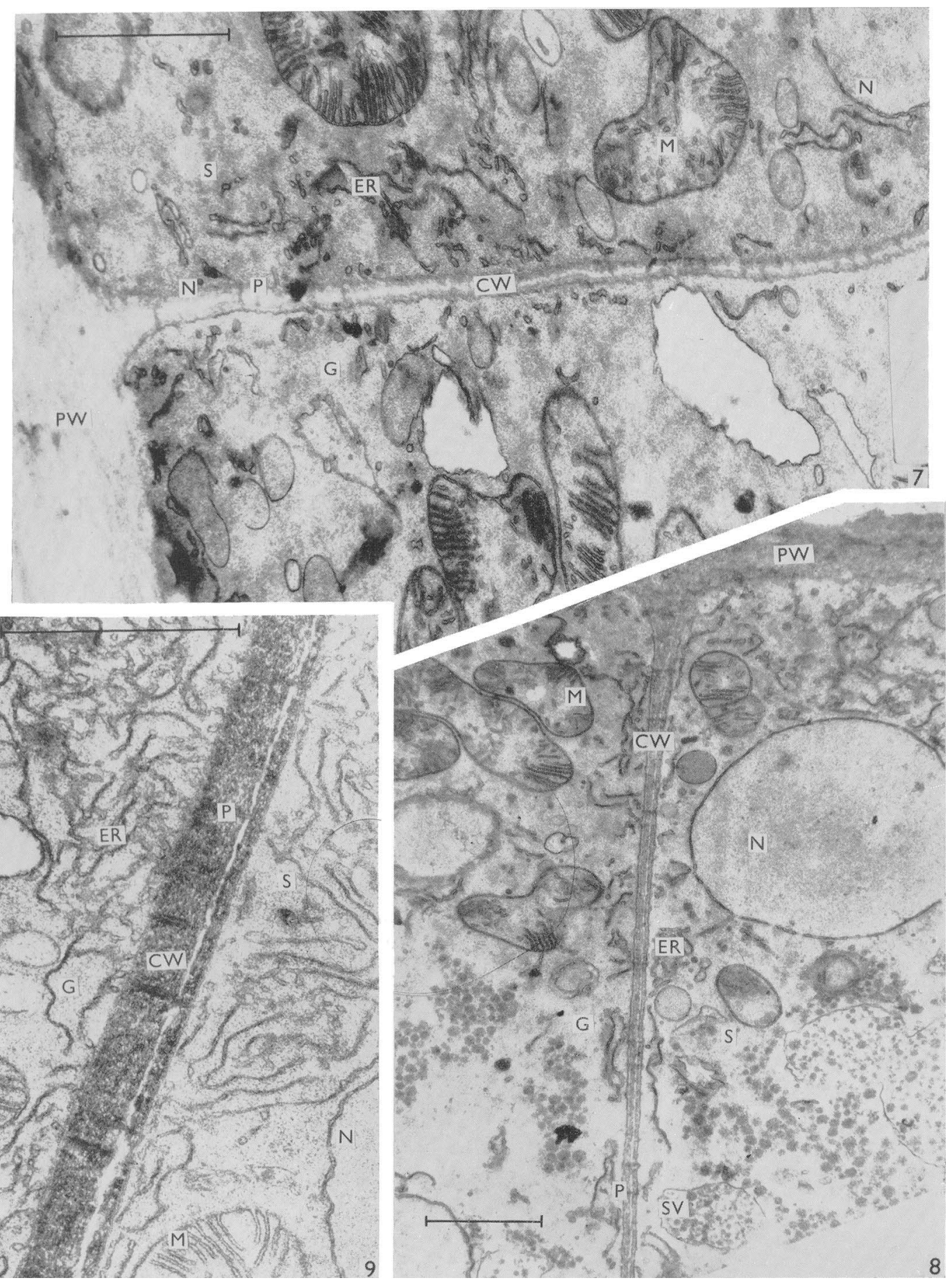

